\title{
Enhanced antidepressant-like effects of the macromolecule trefoil factor 3 by loading into negatively charged liposomes
}

This article was published in the following Dove Press journal:

International Journal of Nanomedicine

12 November 2014

Number of times this article has been viewed

\author{
Jing Qin' \\ Xu Yang ${ }^{1-3}$ \\ Jia $\mathrm{Mi}^{4}$ \\ Jianxin Wang' \\ Jia Hou ${ }^{1,2}$ \\ Teng Shen' \\ Yongji $\mathrm{Li}^{2}$ \\ Bin Wang ${ }^{4}$ \\ Xuri $\mathrm{Li}^{4}$ \\ Weili Zhu ${ }^{5}$ \\ 'Department of Pharmaceutics, School \\ of Pharmacy, Fudan University, Key \\ Laboratory of Smart Drug Delivery, \\ Ministry of Education, Shanghai, \\ ${ }^{2}$ Department of Pharmaceutics, School \\ of Pharmacy, Heilongjiang University \\ of Chinese Medicine, Harbin, \\ ${ }^{3}$ Department of Pharmacy, The Fifth \\ People's Hospital of Shanghai, \\ Fudan University, Shanghai, ${ }^{4}$ Binzhou \\ Medical University, Yantai, ${ }^{5} \mathrm{National}$ \\ Institute on Drug Dependence, Peking \\ University, Beijing, People's Republic \\ of China
}

Correspondence: Weili Zhu National Institute on Drug Dependence, Peking University, 38 Xue Yuan Road, Hai Dian, Beijing I00191, People's Republic of China

Tel +86 I0 82802470

$\mathrm{Fax}+861062032624$

Email zhu_wl@bjmu.edu.cn
Abstract: Immunocytes, mainly neutrophils and monocytes, exhibit an intrinsic homing property, enabling them to migrate to sites of injury and inflammation. They can thus act as Trojan horses carrying concealed drug cargoes while migrating across impermeable barriers to sites of disease, especially the blood-brain barrier (BBB). In this study, to target circulating phagocytic cells, we formulated negatively charged nanosize liposomes and loaded trefoil factor 3 (TFF3) into liposomes by the $\mathrm{pH}$-gradient method. According to the optimized formulation (5:1.5 of lipid to cholesterol, 10:1 of lipid to drug, $10 \mathrm{mg} / \mathrm{mL}$ of lipid concentration, and $10 \mathrm{mmol} / \mathrm{L}$ of phosphate-buffered saline), $44.47 \%$ entrapment efficiency was obtained for TFF3 liposomes with $129.6 \mathrm{~nm}$ particle size and $-36.6 \mathrm{mV}$ zeta potential. Compared with neutrally charged liposomes, the negatively charged liposomes showed a strong binding capacity with monocytes and were effectively carried by monocytes to cross the BBB in vitro. Furthermore, enhanced antidepressant-like effects were found in the tail-suspension and forced-swim tests in mice, as measured by decreased immobility time, as well as increased swimming time and reduced immobility in rats. These results suggested that negatively charged liposomes could improve the behavioral responses of TFF3, and our study opens up a new way for the development of effective therapies for brain disease by increasing the permeability of the BBB.

Keywords: TFF3, drug delivery system, monocytes, brain-targeting drug delivery, antidepressant, blood-brain barrier

\section{Introduction}

Depression is the most common mental disorder, and is increasingly recognized as a public health and social problem worldwide. A total of $4 \%-20 \%$ of the general population will suffer from the disorder some time during their lifetime. ${ }^{1}$ Numerous studies have indicated that depression, as well as other mental illnesses, are closely associated with physical status. ${ }^{2}$ In the early 1990 s, depression was reported for the first time as an illness characterized by cell-mediated immune activation. This observation developed into a novel hypothesis that inflammation and cell-mediated immune activation may be key factors in depression. ${ }^{3}$ Recent evidence suggests that depression is an inflammatory disorder because of the increased production of interleukin (IL)-1 $\beta$, IL-6 and tumor necrosis factor (TNF)- $\alpha$ in people with depression. ${ }^{4}$

Despite breakthroughs in nanotechnology-based approaches, many brain diseases remain undertreated by effective therapies, because of the failure to deliver therapeutic compounds to the brain. ${ }^{5}$ The targeting of drugs within the brain is limited mainly by both the short plasmatic half-life of the therapeutic molecules and by the weak permeability of the blood-brain barrier (BBB). ${ }^{6}$ It was reported that inflammation in 
the brain is found in several conditions, such as psychiatric diseases including depression. ${ }^{7}$ Immunocytes, mainly neutrophils and monocytes, exhibit an intrinsic homing property, enabling them to migrate to sites of injury and inflammation. Therefore, they can act as Trojan horses, carrying concealed drug cargoes while migrating across impermeable barriers to site of disease, especially the BBB. ${ }^{8}$

Using cells as delivery vehicles offers several advantages over common drug-administration regimens, including targeted drug transport and prolonged circulation times, along with reductions in cell and tissue toxicities. ${ }^{9}$ To target circulating phagocytic cells, negatively charged nanosize liposomes were formulated and exhibited an obvious braintargeting drug-delivery effect. ${ }^{10}$ The monocytes were identified as the main carriers for liposomes to the brain. Liposomes are one of the most successful drug-delivery systems, applying nanotechnology to potentiate the therapeutic efficacy and reduce toxicities of conventional medicines. ${ }^{11}$ Liposomes are round bubbles consisting of an aqueous core encapsulated by natural or synthetic phospholipids. This structure turns liposomes into ideal drug carriers. Hydrophilic drugs tend to be entrapped in the core, while hydrophobic ones will be entrapped within the lipid bilayers.

Trefoil factor 3 (TFF3), a member of the TFF family, is stable in the gastrointestinal tract but rapidly eliminated from the circulation. ${ }^{12-14}$ A study showing TFF3 injection induced Fos-positive cells in magnocellular oxytocin neurons in the hypothalamus promoted the research on the role of TFF3 in the central nervous system and related disorders. ${ }^{15}$ In spite of TFF3's potential, the major challenge is its delivery across the $\mathrm{BBB},{ }^{16}$ which has been considered the most important barrier impeding the transport of drugs into the brain. ${ }^{5,17}$ Therefore, an effective drug-delivery system for TFF3 to enhance its stability in plasma and uptake across the BBB has become urgent.

In the present study, negatively charged liposomes loaded with TFF3 (TFF3-Ls) were prepared for targeting the immunocytes in circulating blood, and were then delivered to lesions in the brain. Enhanced antidepressant-like effects were expected. Here, the characteristics, cellular uptake, targeting mechanism, and antidepressant-like effects of TFF3 liposomes are examined.

\section{Materials and methods}

\section{Chemicals and reagents}

DSPE-PEG (1,2-distearoyl-sn-glycero-3-phosphoethanolamine- $N$-[amino(polyethylene-glycol)-2000]) (PEG molecular weight 2,000), distearoyl phosphatidyl glycerol
(DSPG) and soybean phosphatidylcholine (SPC) was from Merck (>99\%; Schaffhausen, Switzerland). Cholesterol, Sephadex G-50, and $N$-formyl-methionyl-leucyl-phenylalanine (fMLP) were from Sigma (St Louis, MO, USA). Recombinant human TFF3 (purity $>98 \%$ ) and TFF3-conjugated cyanine 5 (TFF3-Cy5) were purchased from Beijing Yong Kang Jia Xin Science and Technology Development (Beijing, People's Republic of China [PRC]) and freshly prepared in saline. All other chemicals were of analytical grade.

The human acute monocytic leukemia cell line THP-1 was from the American Type Culture Collection (Rockville, MD, USA), human brain microvessel endothelial cells (HBMECs) were from ScienCell (Carlsbad, CA, USA), and fetal bovine serum and Roswell Park Memorial Institute (RPMI) 1,640 medium were from Invitrogen (Carlsbad, CA, USA).

\section{Cell culture}

HBMECs were cultured on the upper chamber of a Tran$\mathrm{swell}^{\circledR}$ insert of purified collagen with endothelial cell medium containing $1 \%(\mathrm{v} / \mathrm{v})$ endothelial cell-growth supplement, $5 \%(\mathrm{v} / \mathrm{v})$ fetal bovine serum, and $1 \%(\mathrm{v} / \mathrm{v})$ penicillin/ streptomycin solution in a humidified $\mathrm{CO}_{2}$ incubator at $37^{\circ} \mathrm{C} .{ }^{18,19}$ A standard chemotactic factor, $10^{-7} \mathrm{M}$ fMLP was placed in the lower chamber under the HBMEC monolayers to establish a chemotactic gradient for monocyte migration across BBB in vitro. ${ }^{19}$ The THP-1 cell line was maintained in RPMI 1640 media supplemented with 10\% fetal bovine serum at $37^{\circ} \mathrm{C}$ in $95 \%$ humidity and $5 \% \mathrm{CO}_{2}$ atmosphere. ${ }^{20}$

\section{Animals}

Male imprinting control region mice (weighing 18-22 g upon arrival) and male Sprague Dawley rats (weighing 200$220 \mathrm{~g}$ upon arrival) were housed at a constant temperature $\left(23^{\circ} \mathrm{C} \pm 2{ }^{\circ} \mathrm{C}\right)$, in a 12 -hour/12-hour light/dark cycle with free provision of food and water. All of the animal experiments were carried out in compliance with the National Institutes of Health Guide for the Care and Use of Laboratory Animals, and the procedures were approved by the Peking University Animal Use Committee (LA2012/21).

\section{UPLC determination of TFF3}

Ultraperformance liquid chromatography (UPLC) and ultraviolet spectrophotometry were used to determine the concentration of TFF3. The apparatus consisted of an Acquity UPLC ${ }^{\circledR} \mathrm{H}$-class series (Waters, Milford, MA, USA). Chromatographic separation was performed on a $\mathrm{C} 18$ column (Acquity UPLC HSS T3, $1.8 \mu \mathrm{m}, 2.1 \times 100 \mathrm{~mm}$ ) at a detection wavelength of $214 \mathrm{~nm}$. The mobile phase consisted 
of $1 \%$ trifluoroacetic acid (phase A) and 1\%o trifluoroacetic acid-acetonitrile (phase B). The elution profile $(1 \mathrm{~mL} / \mathrm{min})$ was as follows: $0-1$ minutes, linear gradient from $80 / 20$ to $50 / 50$ solvent A/solvent B; $1-2$ minutes, linear gradient from $50 / 50$ to $25 / 75 ; 2-3$ minutes, linear gradient from $25 / 75$ to $80 / 20$ solvent $\mathrm{A} /$ solvent $\mathrm{B}$; $3-5$ minutes, isocratic elution comprised of $80 / 20$ solvent $\mathrm{A} /$ solvent $\mathrm{B}$. The elution rate was at $0.3 \mathrm{~mL} / \mathrm{minute}$ at $30^{\circ} \mathrm{C}$. Calibration standards were made at $10,20,40,80,160,320 \mu \mathrm{g} / \mathrm{mL}$ for TFF3.

\section{Preparation of liposomes}

Liposomes were formulated with SPC, 2 mol\% DSPE-PEG, $1 \mathrm{~mol} \%$ DSPG, and appropriate amounts of cholesterol, and prepared as described previously. ${ }^{21}$ Briefly, a mixture of phospholipids and cholesterol at an appropriate ratio were dissolved in $15 \mathrm{~mL}$ of chloroform. The chloroform was evaporated under a stream of nitrogen gas, and the sample was placed in a high vacuum overnight. The lipid film was rehydrated in $5 \mathrm{~mL}$ of phosphate-buffered saline (PBS; pH 7.4) by gentle mixing and heating. The newly formed multilamellar vesicles were subjected to a $500 \mathrm{~W}$ ultrasonic treatment for 2 minutes by a sonicator (JY92-2D; Xinzhi, Ningbo, Zhejiang, China), and was passed three times through an extruder (LF-1; Avestin, Ottawa, Canada) containing $100 \mathrm{~nm}$ polycarbonate filters. As a result, blank liposomes were obtained.

\section{Remote loading of TFF3 into liposomes}

TFF3 was loaded by the pH-gradient method. Blank liposomes obtained as already mentioned were passed through a Sephadex G-50 column equilibrated with PBS ( $\mathrm{pH} 4.5$ ) to exchange the external buffer (PBS, $\mathrm{pH}$ 7.4). Then, the eluted liposomes had a transmembrane $\mathrm{pH}$ gradient of $\mathrm{pH} 7.4$ inside and $\mathrm{pH} 4.5$ outside. TFF3 was added to the liposome suspension at a modest lipid-to-drug molar ratio and then incubated with liposomes at $37^{\circ} \mathrm{C}$ for 2 hours for remote loading. ${ }^{22}$ To remove the unencapsulated TFF3, the liposome suspensions were passed through a mini-Sephadex G-50 column. Different molar ratios of lipid to drug or lipid to cholesterol, concentration of lipid, and PBS were optimized to obtain a satisfactory entrapment efficiency (EE) and average particle size. The equation for calculating the EE was as follows:

$$
\mathrm{EE}=\mathrm{W}_{\text {interior }} / \mathrm{W}_{\text {total }} \times 100 \% \text {, }
$$

where $\mathrm{W}_{\text {interior }}$ represented the intraliposomal content of $\mathrm{TFF} 3$, and $\mathrm{W}_{\text {total }}$ represented the total content in the liposomal suspension when Triton X-100 was added to the suspension.
A mini-Sephadex G-50 column was used to separate free drug for the calculation of $\mathrm{W}_{\text {interior }}$.

For the preparation of fluorescent liposomes, TFF3-Cy5 was used instead of TFF3 (TFF3-Cy5-Ls). As a control, neutrally charged liposomes loaded with TFF3 (TFF3-NLs) or TFF3-Cy5 (TFF3-Cy5-NLs) without addition of DSPG in the lipid formulation were prepared as mentioned earlier.

\section{Evaluation of characterization of TFF3-Ls}

The negatively charged liposomes (TFF3-Ls, TFF3-Cy5-Ls, and blank liposomes) and neutrally charged liposomes (TFF3-NLs and TFF3-Cy5-NLs) were observed by transmission electron microscopy (JEM-2010F, JEOL, Tokyo, Japan) following negative staining with $1 \%$ uranyl acetate solution. The average particle size and zeta potential of these five liposomes were determined by dynamic light scattering using a Zetasizer Nano ZS (Malvern Instruments, Malvern, UK). The EE of neutrally charged liposomes prepared according to the optimized formulation (SPC instead of DSPG) was assayed to compare with negatively charged liposomes. The stability of TFF3-Ls and TFF3-NLs was evaluated by determining the total TFF3 content in liposomes when Triton100 was added within 12 hours. As a control, the stability of TFF3 in PBS was also evaluated by determining the TFF3 concentration in PBS.

\section{Targeting mechanism investigation}

For studying the binding ability of liposomes with monocytes, THP-1 was used as the model of monocytes. ${ }^{23}$ Different lipid concentrations of TFF3-Cy5-Ls were added to THP-1 for 1 hour's incubation at $37^{\circ} \mathrm{C}$. TFF3-Cy5 solutions of equivalent TFF3 concentrations were added to THP-1. As a control, TFF3-Cy5-NLs without the addition of DSPG were used. After incubation, nonadherent liposomes or TFF3-Cy5 was removed by centrifugation. The stained THP-1 was subjected to an ultrasonic treatment for $\mathrm{Cy} 5$ fluorescence assay with a microplate reader (Thermo Fisher Scientific, Waltham, MA, USA).

For the targeting study in vitro, TFF3-Cy5 solution, TFF3Cy5-NLs, and TFF3-Cy5-Ls were separately added onto HBMECs in the presence of THP-1 cells for 1, 2, or 3 hours' incubation at $37^{\circ} \mathrm{C}$. After incubation, the suspension in the lower chamber was collected and subjected to an ultrasonic treatment for $\mathrm{Cy} 5$ fluorescence assay by microplate reader.

\section{Tail-suspension test in mice}

The tail-suspension test (TST) was performed according to a previous publication. ${ }^{24}$ Briefly, mice were suspended 
$50 \mathrm{~cm}$ above the floor by adhesive tape placed approximately $1 \mathrm{~cm}$ from the tip of the tail. The test was videotaped, and immobility time was measured for 6 minutes. Immobility was defined as the absence of any limb or body movements, with the exception of those caused by respiration, when the mice hung passively and completely motionless. During the test, the mice were separated from each other to prevent visual and acoustic associations. The number of seconds spent immobile was recorded. Observers were blind to the group treatment of the mice.

\section{Forced-swim test in mice}

The forced-swim test (FST) was similar to a previous protocol..$^{25}$ Mice were placed in a $20 \mathrm{~cm}$-diameter $\times 35 \mathrm{~cm}$ height plastic cylinder filled to $20 \mathrm{~cm}$ with $23^{\circ} \mathrm{C}-25^{\circ} \mathrm{C}$ water. The test was videotaped, and immobility time was measured. The definition of immobility was the absence of all movement, with the exception of motions required to maintain the animal's head above the water. The results are expressed as the time spent immobile during the last 4 minutes of the 6 minutes session. Observers were blind to the group treatment of the mice.

\section{Locomotor activity in mice}

Locomotor activity was measured using the open-field test (OFT). Briefly, the apparatus consisted of a $25 \mathrm{~cm}$-diameter $\times 12 \mathrm{~cm}$-height circular arena divided into six equal squares on the floor of the arena. Mice were placed individually in the center of the cage, and the number of crossings to adjacent squares was counted as horizontal locomotor activity, and the total distance traveled ( $\mathrm{mm}$ ) was measured by computer for 5 minutes. Observers were blind to the group treatment of the mice.

\section{Forced-swim test in rats}

The rat FST was similar to a protocol used previously. ${ }^{26,27}$ Briefly, the rats were placed for 15 minutes into a $25 \mathrm{~cm}$ diameter $\times 65$-cm-height plastic cylinder filled to a depth of $45 \mathrm{~cm}$ with $23^{\circ} \mathrm{C}-25^{\circ} \mathrm{C}$ water. The rats were then removed, dried, and returned to their home cage. They were placed again in the cylinders 24 hours later, and a 5-minute swim test was conducted and videotaped. Immobility was defined as the minimum movement required passively to keep the animal's head above the water without other motions. Climbing was defined as the upward-directed movement of the forepaws against the wall. The results are expressed as the time (in seconds) that the animals spent immobile during the 5-minute test.

\section{Locomotor activity in rats}

Locomotor activity in rats was measured in the OFT. Briefly, the open-field apparatus is a square arena consisting of a $75 \times 75 \times 40 \mathrm{~cm}$ square arena that was divided into 25 equal squares $(15 \times 15 \mathrm{~cm})$ on the floor. Each rat was placed in the center of the cage, and the number of crossings (entering the adjacent square line) was counted for 5 minutes to assay the locomotor activity.

\section{Results UPLC assay}

UPLC images of TFF3-Ls and blank liposomes (Figure 1) show that the endogenous interferences had no effect on the separation of TFF3. The calibration curves for TFF3 were linear from 10 to $320 \mu \mathrm{g} / \mathrm{mL}$ ( $r=0.9997$ ), with overall mean recoveries above $97.6 \%$. The equation for TFF3 was $y=0.0004 x-0.2886$, where $y$ represented concentration of TFF3 and $x$ represented peak area. Therefore, the quantification of TFF3 was performed under the UPLC condition.

\section{Optimization of TFF3-Ls}

To obtain high EE, the influence of the different formulation factors, including the ratio of lipid to drug and lipid to cholesterol, and concentration of lipid and PBS were investigated. The ratio of lipid to cholesterol of 5:1.5 (Figure 2A) obtained the highest EE, with a $150 \mathrm{~nm}$ particle size among three groups. A significant decrease in EE was observed when the ratio of lipid to cholesterol was at $5: 1$ or 5:2 compared with $5: 1.5$. This might have resulted from the influence of cholesterol on the fluidity of the lipid bilayer. Therefore, a

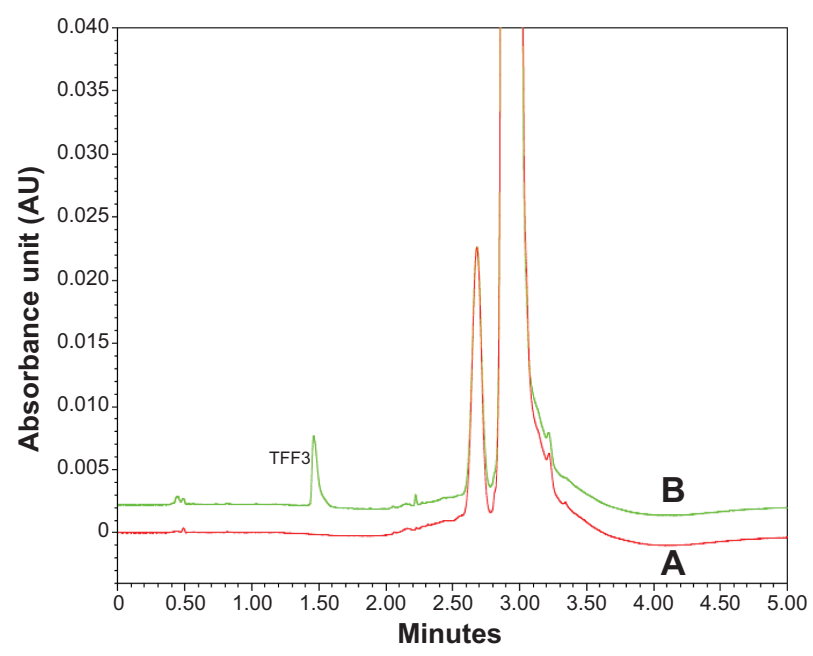

Figure I Representative ultraperformance liquid chromatogram of blank liposomes (A) and TFF3-Ls (B).

Abbreviation: TFF3-Ls, trefoil factor 3-loaded negatively charged liposomes. 
A
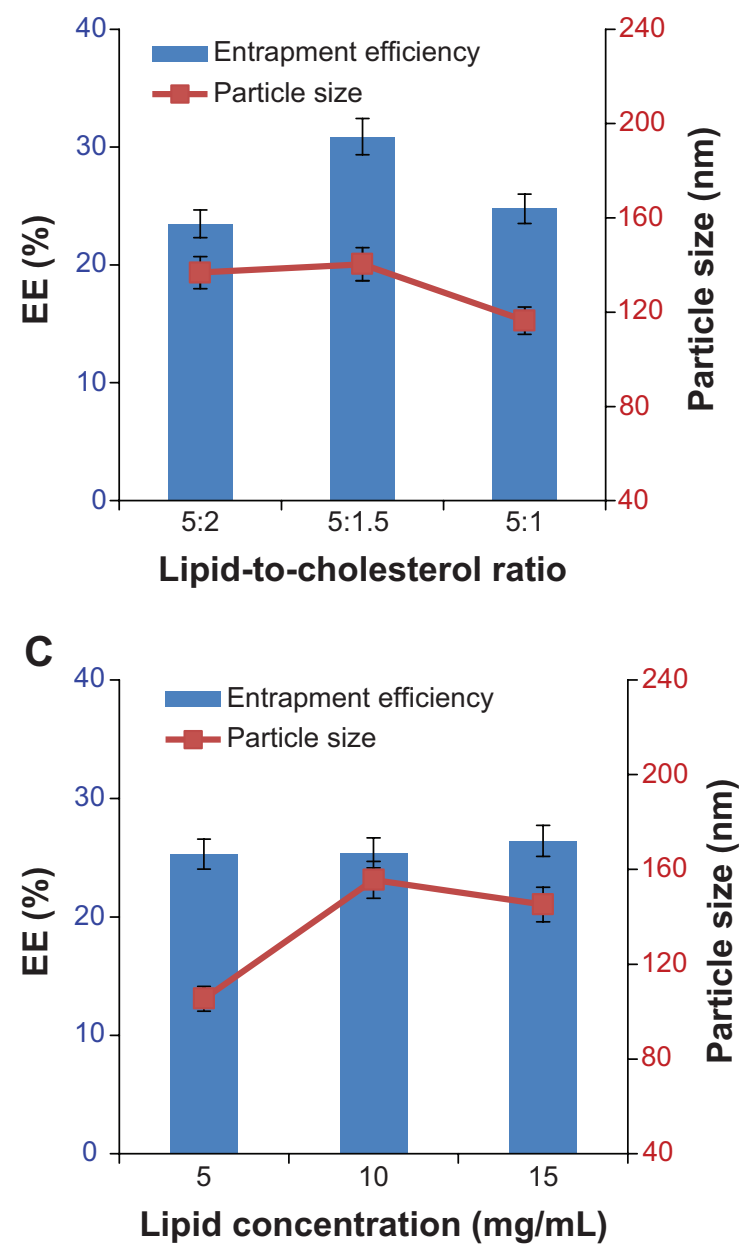

B
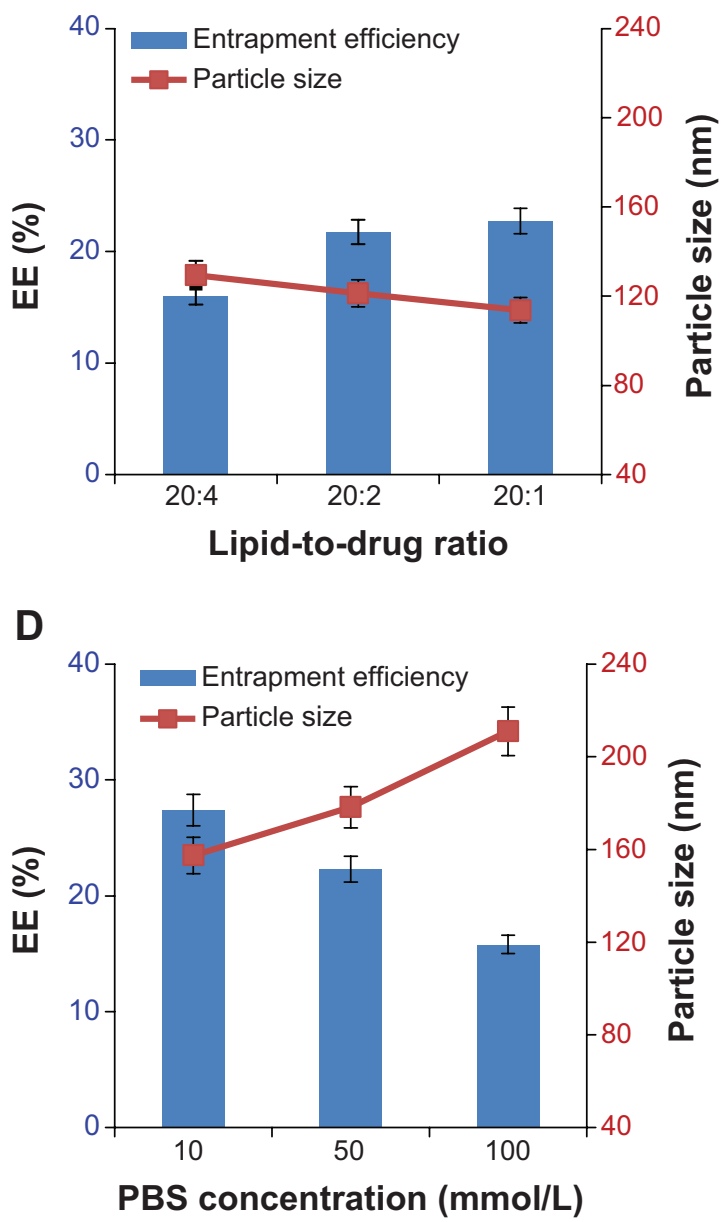

Figure 2 Influence of the different formulation factors on entrapment efficiency (EE) and particle size.

Notes: (A) The ratio of lipid to cholesterol. (B) The ratio of lipid to drug. (C) The lipid concentration. (D) PBS concentration.

Abbreviation: PBS, phosphate-buffered saline.

modest content of cholesterol is necessary for the lipid formulation. ${ }^{28}$ Considering $150 \mathrm{~nm}$ particle size was suitable for uptake by monocyte, ${ }^{9}$ the ratio of $5: 1.5$ lipid to cholesterol was chosen in the present study.

The EE exhibited a tendency to increase with the rising of lipid to drug, while the average particle size did not show a significant change. There was no significant difference in $\mathrm{EE}$ between 20:1 and 20:2. Considering the loading efficiency, the ratio of 20:2 was preferred (Figure 2B).

The concentration of lipid had no effect on the EE but significantly influenced the average particle size (Figure 2C). About $150 \mathrm{~nm}$ average particle size was obtained when the concentration of lipid was up to $10-15 \mathrm{mg} / \mathrm{mL}$ while a $105 \mathrm{~nm}$ particle size was obtained at $5 \mathrm{mg} / \mathrm{mL}$. High lipid concentration would have led to an aggregation of liposome, which resulted in an increase in particle size. Given the possibility that precipitation might occur at high lipid concentration, $10 \mathrm{mg} / \mathrm{mL}$ lipid concentration was preferred.
The high concentration of PBS induced an obvious instability in particle size (rising up to $200 \mathrm{~nm}$ ) and decrease in EE (Figure 2D). As such, $10 \mathrm{mmol} / \mathrm{L}$ of PBS concentration was adopted in the formulation. Taken together, 5:1.5 lipid to cholesterol, 10:1 lipid to drug, $10 \mathrm{mg} / \mathrm{mL}$ lipid concentration, and $10 \mathrm{mmol} / \mathrm{L}$ PBS were used in the final formulation. According to the optimized formulation, $44.47 \%$ EE was obtained for TFF3-Ls.

\section{Characteristics of TFF3 liposomes}

The average size and zeta potential of negatively charged liposomes (TFF3-Ls, TFF3-Cy5-Ls, and blank liposomes) and neutrally charged liposomes (TFF3-NLs and TFF3-Cy5NLs) are shown in Table 1. There was no obvious difference in average size for TFF3-Ls, TFF3-NLs, TFF3-Cy5-Ls, TFF3-Cy5-NLs, or blank liposomes. The zeta potential of negatively charged liposomes was much lower than that of neutrally charged liposomes, resulting from the addition 
Table I The characteristics of various TFF3 liposomes

\begin{tabular}{llllll}
\hline & Blank liposome & TFF3-Ls & TFF3-NLs & TFF3-Cy5-Ls & TFF3-Cy5-NLs \\
\hline Particle size $(\mathrm{nm})$ & I36.7 (PDI 0.16I) & I29.6 (PDI 0.185) & I30.6 (PDI 0.I I2) & I27.3 (PDI 0.132) & I38.5 (PDI 0.1 I7) \\
Zeta potential (mV) & -32.7 & -36.6 & -12.9 & -34.5 & -15.6 \\
EE $(\%)$ & - & 44.47 & 44.23 & 42.16 & 43.26 \\
\hline
\end{tabular}

Abbreviations: TFF3-Cy5-Ls, trefoil factor 3-cyanine 5-loaded negatively charged liposomes; TFF3-Cy5-NLs, TFF3-Cy5-loaded neutrally charged liposomes; EE, entrapment efficiency; PDI, polydispersity index; TFF3-Ls, negatively charged liposomes loaded with TFF3; TFF3-NLs, neutrally charged liposomes loaded with TFF3.

of DSPG in lipid formula. The representative electron micrographs of negatively charged liposomes and neutrally charged liposomes are shown in Figure 3. Large unilamellar vesicles were observed in micrographs. The EE did not show any significant differences among those five TFF3 liposomes. Therefore, the loading of TFF3 and the addition of fluorescence lipid or DSPG had no influence on modality, EE, or particle size of liposomes.

In the stability study, TFF3 concentration showed no significant change for either TFF3-Ls or TFF3-NLs within 12 hours (Figure 3F). Compared with the TFF3 solution, the stability of TFF3 was greatly enhanced by loading into negatively charged liposomes or neutrally charged liposomes. As a result, satisfactory negatively charged liposomes were obtained in this study.

\section{Targeting mechanism investigation}

As shown in Figure 4, the fluorescence strength of TFF3Cy5-Ls was the strongest among the three groups and increased with concentration. It suggested that the binding of TFF3-Cy5-Ls with monocytes was significantly stronger than that of TFF3-Cy5-NLs. TFF3-Cy5 did not bind with monocytes. Negatively charged liposomes greatly enhanced the binding capacity of liposomes with monocytes. Furthermore, the fluorescence strength of migrated TFF3-Cy5-Ls was the strongest among those groups after 3 hours' incubation (Figure 5). This suggested that negatively charged liposomes carried macromolecular drug across the BBB because of the high binding capacity with monocytes. TFF3-Cy5 could not cross the BBB regardless of whether it was by itself or in the presence of THP-1.

\section{Antidepressant-like effects of acute TFF3 administration in mice}

Mice receiving saline, TFF3, TFF3-NLs, or TFF3-Ls were injected (intraperitoneally) 30 minutes before the TST ( $\mathrm{n}=8$ per group), respectively. Compared with the saline group, significant change was observed for both TFF3-Ls
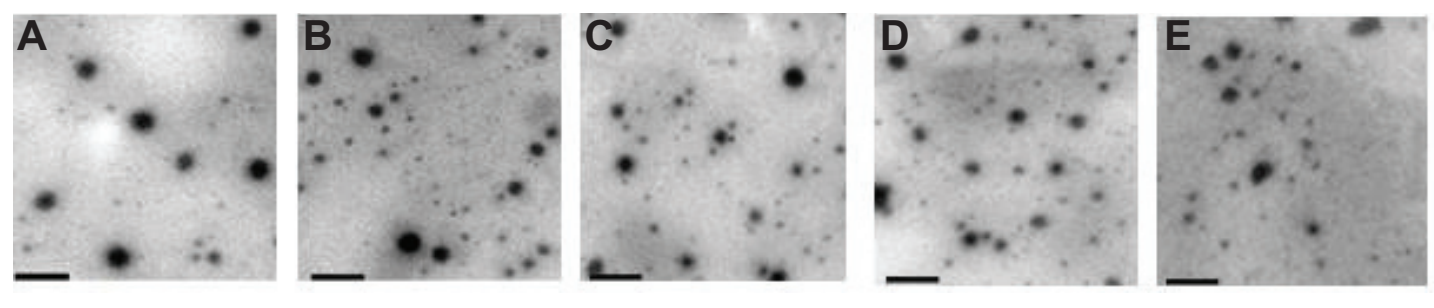

\section{$\mathbf{F}$}

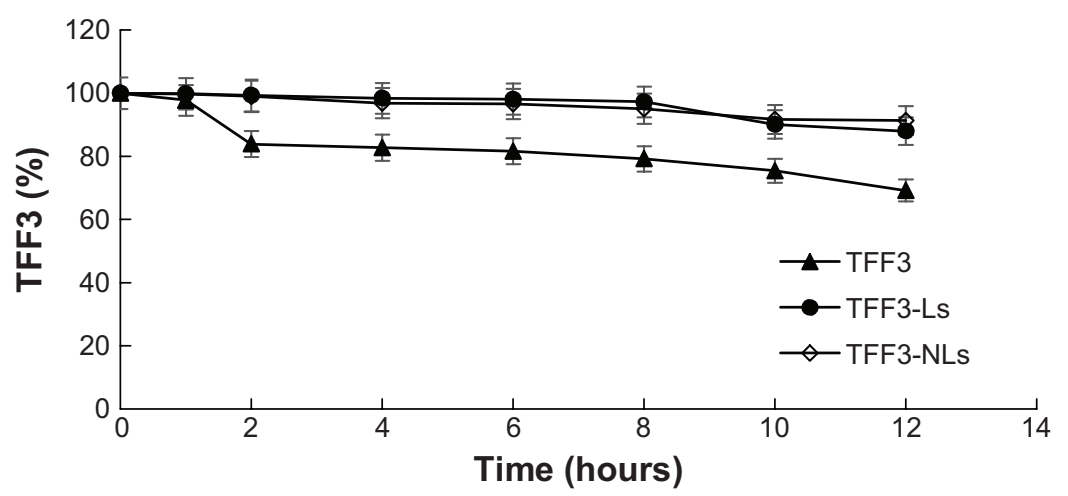

Figure 3 Electron micrographs (A-E) and stability (F) of TFF3 liposomes.

Notes: (A) Blank liposomes; (B) TFF3-Ls; (C) TFF3-NLs; (D) TFF3-Cy5-Ls; (E) TFF3-Cy5-NLs. Scale bar represents $200 \mathrm{~nm}$.

Abbreviations: TFF3-Cy5-Ls, trefoil factor 3-cyanine 5-loaded negatively charged liposomes; TFF3-Cy5-NLs, TFF3-Cy5-loaded neutrally charged liposomes; TFF3-Ls, negatively charged liposomes loaded with TFF3; TFF3-NLs, neutrally charged liposomes loaded with TFF3. 


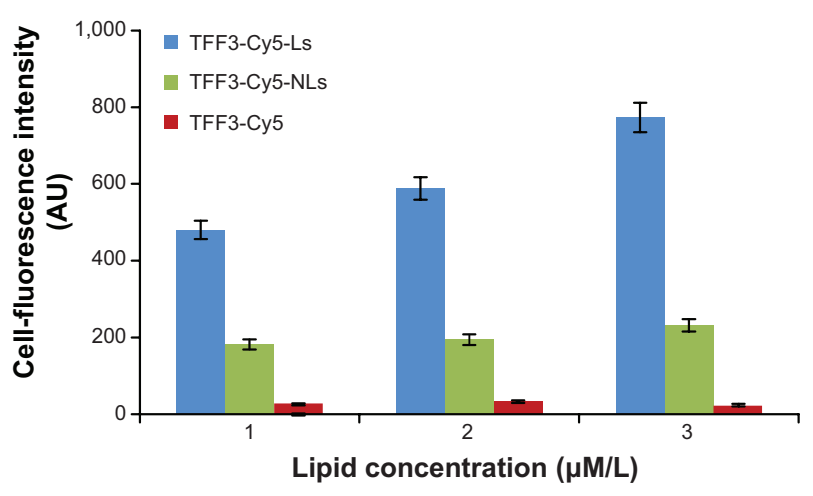

Figure 4 Binding ability of TFF3-Cy5, TFF3-Cy5-Ls, and TFF3-Cy5-NLs with monocytes.

Abbreviations: TFF3-Cy5-Ls, trefoil factor 3-cyanine 5-loaded negatively charged liposomes; TFF3-Cy5-NLs, TFF3-Cy5-loaded neutrally charged liposomes; TFF3-Cy5, TFF3-conjugated cyanine 5 .

$(P<0.001)$ and TFF3-NLs $(P<0.01)$, while the TFF3 solution showed no significant change. The data showed that TFF3-NLs $(P<0.01)$ and TFF3-Ls $(P<0.05)$ produced stronger antidepressant-like effects compared with the TFF3 solution, as measured by the decreased immobility time. TFF3-Ls $(P<0.01)$ was more effective than TFF3-NLs (Figure 6, A and B).

Additionally, acute TFF3-NL $(P<0.01)$ and TFF3-L $(P<0.001)$ administration also reduced the immobility time in the FST compared with the saline group, while no significant change was observed for the TFF3 solution. Furthermore, TFF3-Ls were more effective than TFF3-NLs (Figure 6, C and D). However, TFF3, TFF3-NL, or TFF3-L treatment did not affect the normal locomotion, as indicated by crossings (Figure 6, E and F) and total distance traveled in the OFT (Figure 6G).

We further determined the potential antidepressantlike effect of TFF3, TFF3-NLs, and TFF3-Ls in rats. Four groups of rats ( $\mathrm{n}=8$ per group) were treated with saline,

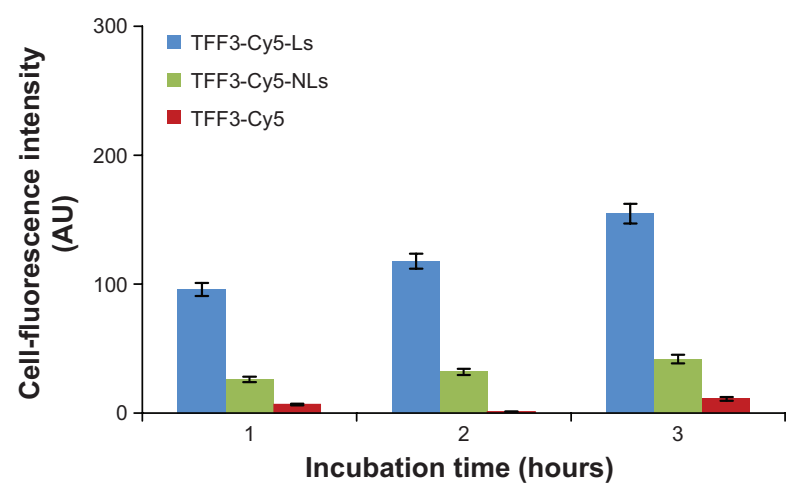

Figure 5 Amount of migrated TFF3-Cy5, TFF3-Cy5-Ls, and TFF3-Cy5-NLs with monocytes after different incubation times.

Abbreviations: TFF3-Cy5-Ls, trefoil factor 3-cyanine 5-loaded negatively charged liposomes; TFF3-Cy5-NLs, TFF3-Cy5-loaded neutrally charged liposomes; TFF3-Cy5, TFF3-conjugated cyanine 5 .
TFF3, TFF3-NLs, or TFF3-Ls 30 minutes before the FST (Figure 7A). The analysis data showed that acute TFF3-NL $(P<0.05)$ and TFF3-L $(P<0.01)$ administration significantly decreased immobility time in the FST compared with the saline group, and no significant decrease in immobility time was observed for the TFF3 solution. TFF3-Ls were more effective than TFF3-NLs (Figure 7B). We also found that acute TFF3-NL $(P<0.001)$ and TFF3-L $(P<0.001)$ administration significantly increased swimming time in the FST, and the TFF3 solution showed no effect on swimming time. The antidepressant-like effects of TFF3-Ls $(P<0.01)$ was stronger than TFF3-NLs (Figure 7B). The TFF3 solution had no influence on swimming time. Moreover, four separate groups of rats treated with saline, TFF3, TFF3-NLs, or TFF3-Ls were used to measure locomotor activity in rats (Figure 7D). The results showed that acute TFF3, TFF3-NLs, and TFF3-Ls did not affect normal locomotion, reflected by the number of crossings in the OFT (Figure 7E). This suggested that reductions in immobility and increases in swimming were not attributable to alterations in locomotor activity. These results suggest that acute TFF3 solution administration did not produce an antidepressant effect; the antidepressant behavioral effects of TFF3 were enhanced by loading into liposomes; negatively charged liposomes showed more effective antidepressant effect resulting from the obvious brain-targeting effect.

\section{Discussion}

It is now known that the brain is under immunological surveillance, and thus phagocytic cells of the innate immune system, mainly neutrophils and monocytes, can be exploited as transporters of drugs to the brain. In a previous study, monocytes were believed to be the main contributor in this targeting drug-delivery system. ${ }^{10}$ Therefore, the main aim of this study was to enhance the affinity of liposomes with monocytes.

It has been reported that negatively charged liposomes that are not of a ultrasmall size will result in efficient uptake by phagocyte cells (monocytes and neutrophils). These liposomes are rapidly cleared by blood monocytes, neutrophils, and macrophages of the liver, spleen, and bone marrow. Drug was transported across the BBB by the phagocyte cells and subsequently released from these cells in the brain. ${ }^{10}$ Therefore, negatively charged liposomes with proper particle size were the key factors in this targeting drug-delivery system. In the present study, a negatively charged liposome of about $130 \mathrm{~nm}$ particle size was obtained. Comparing with neutrally charged liposomes, they showed a greatly higher 
A

Timeline (minutes)

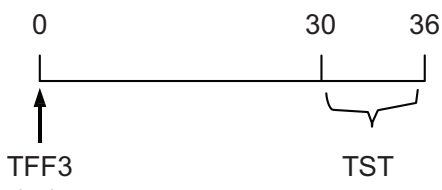

(IP)

B

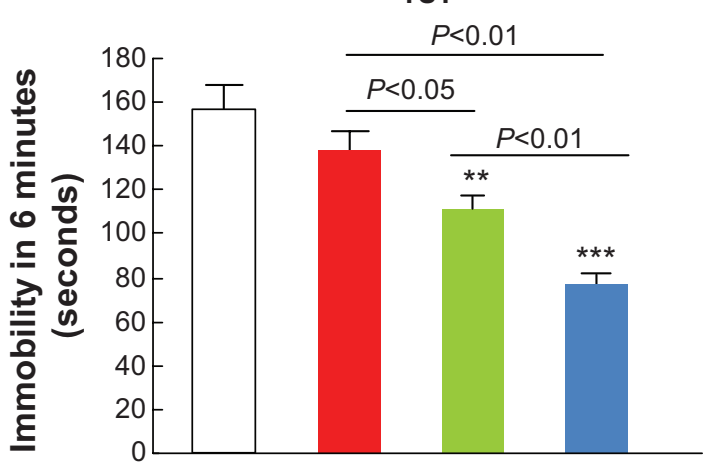

C Timeline (minutes)

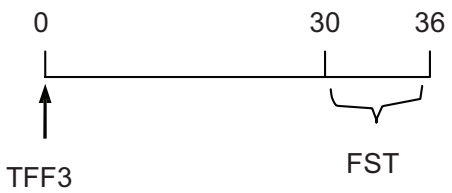

(IP)
D

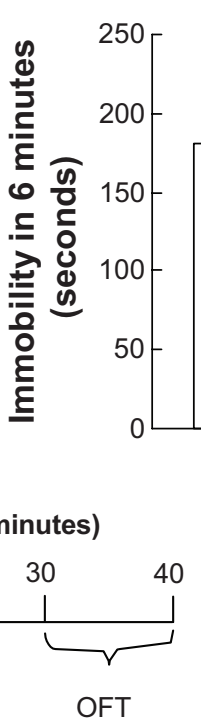

$\square$ Saline

FST

TFF3 solution

TFF3-NLs

TFF3-Ls

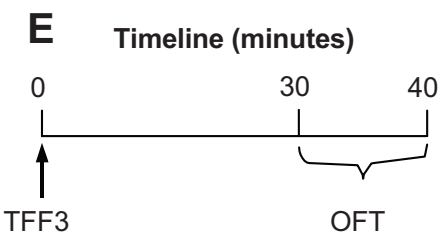

(IP)

F

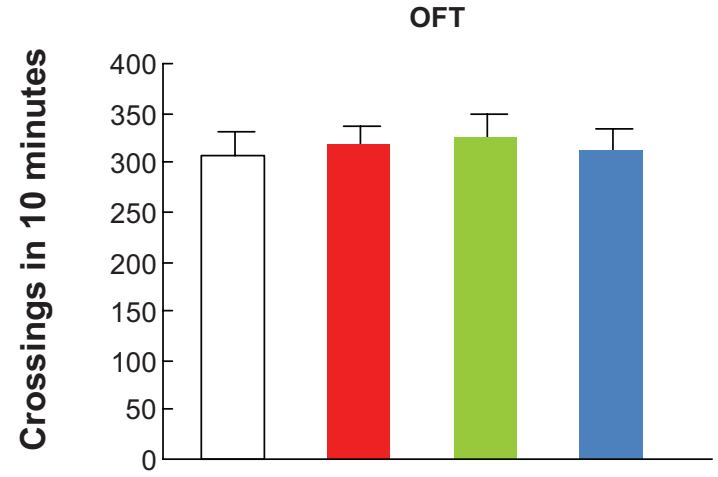

G

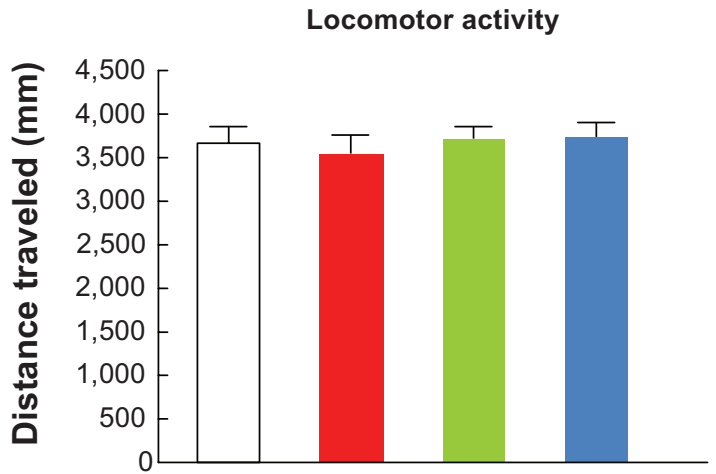

Figure 6 The antidepressant-like effect of acute TFF3 administration in mice.

Notes: (A) Experimental procedure for the tail-suspension test (TST). The TSTs were conducted 30 minutes after a single injection of saline, TFF3, TFF3-NLs, or TFF3-Ls (intraperitoneally [IP]). (B) Acute TFF3-L and TFF3-NL administration significantly decreased immobility time in the TST. (C) Experimental procedure for the forced-swim test (FST). (D) Acute TFF3-L and TFF3-NL administration significantly reduced the immobility time in the FST. (E) Experimental procedure for the open-field test (OFT). (F) Acute TFF3 solution, TFF3-NL, or TFF3-L administration had no effects on crossings in the OFT. (G) Acute TFF3 solution, TFF3-NLs, or TFF3-Ls did not affect locomotor activity as measured by total distance traveled in mice either. The OFT was monitored for 10 minutes. Data are expressed as means \pm standard error of the mean ( $\mathrm{n}=8$ per group). $* * P<0.01 ; * * * P<0.001$.

Abbreviations: TFF3-Ls, trefoil factor 3-loaded negatively charged liposomes; TFF3-NLs, TFF3-loaded neutrally charged liposomes; TFF3, trefoil factor 3.

binding ability with monocytes and obtained an obvious migration across the BBB in vitro and in vivo.

Incorporation of liposomes into protein formulations for drug-delivery systems may represent an attractive method for sustained release, reducing the degradation and enhancing the pulmonary permeation rate. ${ }^{22}$ The engineering of an effective liposomal drug formulation for drug delivery must take into consideration encapsulation efficiency, vesicle stability, and the leakage problem associated with the delivery process. ${ }^{29}$ The use of drug-containing liposomes in therapeutics requires 


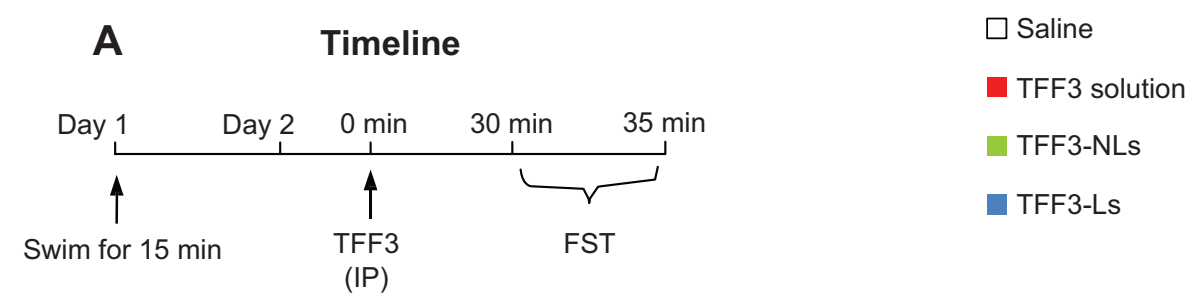

B

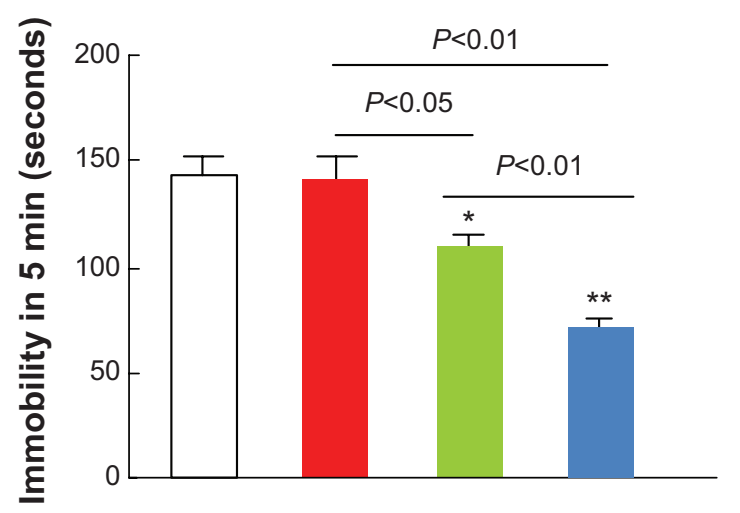

D

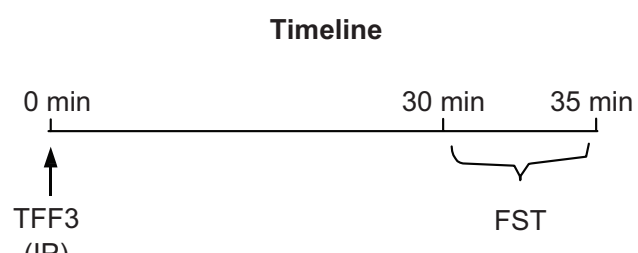

C

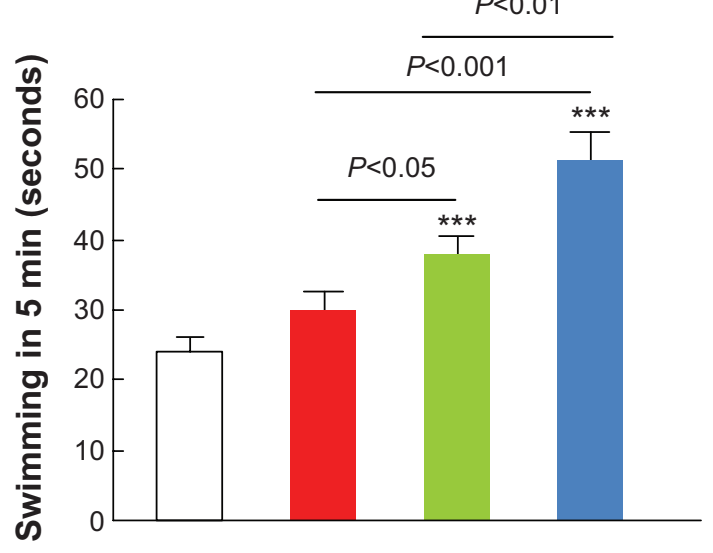

E

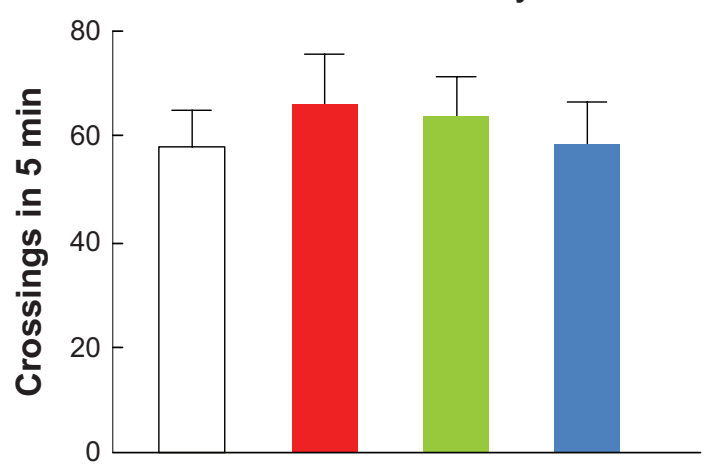

Figure 7 The antidepressant-like effect of acute TFF3 administration in rats.

Notes: (A) Experimental procedure for the forced-swim test (FST). The FSTs were conducted 30 minutes after a single injection of saline, TFF3, TFF3-NLs, or TFF3-Ls (intraperitoneally [IP]). (B) Acute TFF3-L and TFF3-NL administration significantly decreased immobility time in the FST. (C) Acute TFF3-L and TFF3-NL administration significantly increased the swimming time in the FST. (D) Experimental procedure for the open-field test. (E) Acute TFF3 solution and TFF3 liposome administration had no effects on crossings in the OFT. Data are expressed as means \pm standard error of the mean ( $\mathrm{n}=8$ per group). $* \mathrm{P}<0.05 ; * * \mathrm{P}<0.0 \mathrm{I} ;{ }^{* * *} \mathrm{P}<0.00 \mathrm{I}$.

Abbreviations: TFF3-Ls, trefoil factor 3-loaded negatively charged liposomes; TFF3-NLs, TFF3-loaded neutrally charged liposomes; min, minutes; TFF3, trefoil factor 3.

techniques of effective drug loading in vesicles that retain their stability in the biological milieu and exhibit optimal body-distribution profiles. The dehydration-rehydration method and pH-gradient method were often used, followed or not by repeated extrusion. ${ }^{16}$ In the present study, TFF3 was efficiently loaded into preformed liposomes and high EE was obtained by the $\mathrm{pH}$-gradient method. Furthermore, liposomes significantly enhanced the stability of TFF3, which might contribute to targeting delivery and the increased antidepressant-like effect.

THP-1 cells have become one of most widely used cell lines to investigate the function and regulation of monocytes. The interactions between THP-1 cells and various vascular cells (such as endothelial cells, smooth-muscle cells, 
adipocytes, and $\mathrm{T}$ cells) provide insight into the roles of the interconnection of monocytes and macrophages with other vascular cells during vascular inflammation. ${ }^{23}$ Technically, THP-1 cells have some advantages over human primary monocytes and macrophages. Their homogeneous genetic background minimizes the degree of variability in the cell phenotype. This trait is particularly important when studying the biological function of chemicals with high variability. Furthermore, THP-1 cells can be stored indefinitely in liquid nitrogen. Following an appropriate procedure, this cell line can be recovered without obvious detrimental effect on monocyte/macrophage features or cell viability. This ability to store cells guarantees sufficient cells for deoxyribonucleic acid, ribonucleic acid, biometal, and protein studies. In contrast, the availability of primary human monocytes and macrophages is limited, accounting for only 3\%-9\% of all leukocytes. ${ }^{30,31}$ Therefore, in this study, THP-1 was very suitable to study the combination of monocytes with liposomes. HBMECs are a well-established in vitro model of the human $\mathrm{BBB}$ that constitutively expresses adhesion molecules relative to monocyte migration across the $\mathrm{BBB}$. The chemotactic gradient of fMLP was used to attract monocytes to migrate across the BBB. ${ }^{19}$ As a result, HBMECs and THP-1 were used as the model to investigate the targeting effect of liposomes with monocytes crossing the BBB.

The TST and FST are two widely used behavioral models in rodents used to assess putative antidepressant compounds. The increased immobility time in animals reflects the desperate behavior usually seen in depressed patients. Medications that can reduce the immobility time in the TST and FST reflect a potential antidepressant-like activity, such as conventional antidepressant drugs and electroconvulsive therapy. The increased swimming time that animals spend in the FST indicates the ability to escape actively from stressful events. Therefore, the score of swimming time was another useful indicator for the therapeutic benefits of possible agents in the treatment of depression and stress-related disorder. We found here that TFF3-Ls decreased immobility in both the TST and FST in mice, and increased swimming time in the FST of rats, suggesting that TFF3-Ls could be used for the therapy of behavioral dysfunctions in a model of desperate animals. Furthermore, in the present study, to exclude the possibility that the antidepressant-like effects of TFF3-NLs and TFF3-Ls were attributable to stimulatory effects on locomotor function, we evaluated TFF3-induced spontaneous activity in the OFT. The results showed that mice treated with TFF3-NLs and TFF3-Ls exhibited no alterations in spontaneous activity in the OFT, indicating that the decreases in immobility in the FST and TST were induced by increases in active movements, such as struggling and swimming, but not caused by motor stimulation. Furthermore, our data also showed that acute systemic TFF3-L administration exerted stronger antidepressant-like properties than TFF3-NLs in the rat FST and in the mouse TST and FST, as reflected by decreased immobility and increased swimming time, suggesting that negatively charged liposomes enhanced the effective delivery of TFF3 solution across BBB.

\section{Conclusion}

In the present study, negatively charged liposomes were prepared by the addition of DSPG, and a high EE of TFF3 was obtained by a remote-loading method. The TFF3-Ls showed an excellent ability to migrate across the BBB as a result of high binding efficiency with THP-1. Subsequently, an enhanced antidepressant-like effect of TFF3 loaded into negatively charged liposomes was obtained in animal models of depression. These findings provide prospective insights into the development of TFF3-Ls as a potential antidepressant drug relative to clinically used antidepressants.

\section{Acknowledgments}

This work was supported by the National Natural Science Foundation of China (81102386, 81371489); the Beijing Higher Education Young Elite Teacher Project (YETP0068); the National Science and Technology Major Project (2012ZX09103-301-049); the Specialized Research Fund for the Doctoral Program of Higher Education (20110071120042); the School of Pharmacy, Fudan University and the Open Project Program of Key Lab of Smart Drug Delivery (Fudan University), Ministry of Education, PRC; the Program for Scientific Research Innovation team in Colleges and Universities of Shandong Province and Taishan Scholars Construction Engineering; and the National Key Basic Research Program of China (program 973, 2013CB932502).

\section{Disclosure}

The authors report no conflicts of interest in this work.

\section{References}

1. Bakish D. New standard of depression treatment: remission and full recovery. J Clin Psychiatry. 2001;62 Suppl 26:5-9.

2. Richardson LP, Lozano P, Russo J, McCauley E, Bush T, Katon W. Asthma symptom burden: relationship to asthma severity and anxiety and depression symptoms. Pediatrics. 2006;118(3):1042-1051.

3. Jiang M, Qin P, Yang X. Comorbidity between depression and asthma via immune-inflammatory pathways: a meta-analysis. $J$ Affect Disord. 2014;166:22-29. 
4. Maes M. Depression is an inflammatory disease, but cell-mediated immune activation is the key component of depression. Prog Neuropsychopharmacol Biol Psychiatry. 2011;35(3):664-675.

5. Chen Y, Liu L. Modern methods for delivery of drugs across the bloodbrain barrier. Adv Drug Deliv Rev. 2012;64(7):640-665.

6. Beduneau A, Hindre F, Clavreul A, Leroux JC, Saulnier P, Benoit JP. Brain targeting using novel lipid nanovectors. J Control Release. 2008; 126(1):44-49.

7. Bailey MT, Kinsey SG, Padgett DA, Sheridan JF, Leblebicioglu B. Social stress enhances IL- $1 \beta$ and TNF- $\alpha$ production by Porphyromonas gingivalis lipopolysaccharide-stimulated CD11b+ cells. Physiol Behav. 2009;98(3):351-358.

8. Haney MJ, Zhao Y, Li S, et al. Cell-mediated transfer of catalase nanoparticles from macrophages to brain endothelial, glial and neuronal cells. Nanomedicine (Lond). 2011;6(7):1215-1230.

9. Batrakova EV, Gendelman HE, Kabanov AV. Cell-mediated drug delivery. Expert Opin Drug Deliv. 2011;8(4):415-433.

10. Afergan E, Epstein H, Dahan R, et al. Delivery of serotonin to the brain by monocytes following phagocytosis of liposomes. J Control Release. 2008;132(2):84-90.

11. Fan Y, Zhang Q. Development of liposomal formulations: from concept to clinical investigations. Asian J Pharm Sci. 2013;8(2):81-87.

12. Thim L, Woldike HF, Nielsen PF, Christensen M, Lynch-Devaney K, Podolsky DK. Characterization of human and rat intestinal trefoil factor produced in yeast. Biochemistry. 1995;34(14):4757-4764.

13. Poulsen SS, Thulesen J, Nexo E, Thim L. Distribution and metabolism of intravenously administered trefoil factor 2/porcine spasmolytic polypeptide in the rat. Gut. 1998;43(2):240-247.

14. Kjellev S, Vestergaard EM, Nexo E, et al. Pharmacokinetics of trefoil peptides and their stability in gastrointestinal contents. Peptides. 2007; 28(6):1197-1206.

15. Derbyshire A, Ludwig M. TFF3 induced Fos protein expression in the magnocellular oxytocin neurons of the hypothalamus. Peptides. 2004; 25(5):833-838.

16. Brasnjevic I, Steinbusch HW, Schmitz C, Martinez-Martinez P. Delivery of peptide and protein drugs over the blood-brain barrier. Prog Neurobiol. 2009;87(4):212-251.

17. Neuwelt E, Abbott NJ, Abrey L, et al. Strategies to advance translational research into brain barriers. Lancet Neurol. 2008;7(1):84-96.

18. Kuo YC, Lu CH. Effect of human astrocytes on the characteristics of human brain-microvascular endothelial cells in the blood-brain barrier. Colloids Surf B Biointerfaces. 2011;86(1):225-231.

19. Wong D, Prameya R, Dorovini-Zis K. Adhesion and migration of polymorphonuclear leukocytes across human brain microvessel endothelial cells are differentially regulated by endothelial cell adhesion molecules and modulate monolayer permeability. J Neuroimmunol. 2007;184(1-2):136-148.
20. Jonsdottir G, Ingolfsdottir IE, Thormodsson FR, Petersen PH. Endogenous aggregates of amyloidogenic cystatin $\mathrm{C}$ variant are removed by THP-1 cells in vitro and induce differentiation and a proinflammatory response. Neurobiol Aging. 2013;34(5):1389-1396.

21. Dos Santos N, Cox KA, McKenzie CA, et al. pH gradient loading of anthracyclines into cholesterol-free liposomes: enhancing drug loading rates through use of ethanol. Biochim Biophys Acta. 2004; 1661(1):47-60.

22. Hwang SH, Maitani Y, Qi XR, Takayama K, Nagai T. Remote loading of diclofenac, insulin and fluorescein isothiocyanate labeled insulin into liposomes by $\mathrm{pH}$ and acetate gradient methods. Int J Pharm. 1999; 179(1):85-95.

23. Qin Z. The use of THP-1 cells as a model for mimicking the function and regulation of monocytes and macrophages in the vasculature. Atherosclerosis. 2012;221(1):2-11.

24. Steru L, Chermat R, Thierry B, Simon P. The tail suspension test: a new method for screening antidepressants in mice. Psychopharmacology (Berl). 1985;85(3):367-370.

25. Porsolt RD, Le Pichon M, Jalfre M. Depression: a new animal model sensitive to antidepressant treatments. Nature. 1977;266(5604):730-732.

26. Porsolt RD, Anton G, Blavet N, Jalfre M. Behavioural despair in rats: a new model sensitive to antidepressant treatments. Eur J Pharmacol. 1978;47(4):379-391.

27. Lucki I. The forced swimming test as a model for core and component behavioral effects of antidepressant drugs. Behav Pharmacol. 1997; 8(6-7):523-532.

28. Zucker D, Marcus D, Barenholz Y, Goldblum A. Liposome drugs' loading efficiency: a working model based on loading conditions and drug's physicochemical properties. J Control Release. 2009;139:73-80.

29. Wang CH, Huang YY. Encapsulating protein into preformed liposomes by ethanol-destabilized method. Artif Cells Blood Substit Immobil Biotechnol. 2003;31(3):303-312.

30. Rogers PD, Thornton J, Barker KS, et al. Pneumolysin-dependent and -independent gene expression identified by cDNA microarray analysis of THP-1 human mononuclear cells stimulated by Streptococcus pneumoniae. Infect Immun. 2003;71(4):2087-2094.

31. Cousins RJ, Blanchard RK, Popp MP, et al. A global view of the selectivity of zinc deprivation and excess on genes expressed in human THP-1 mononuclear cells. Proc Natl Acad Sci U S A. 2003;100(12) 6952-6957.
International Journal of Nanomedicine

\section{Publish your work in this journal}

The International Journal of Nanomedicine is an international, peerreviewed journal focusing on the application of nanotechnology in diagnostics, therapeutics, and drug delivery systems throughout the biomedical field. This journal is indexed on PubMed Central,

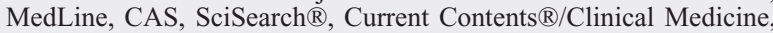

\section{Dovepress}

Journal Citation Reports/Science Edition, EMBase, Scopus and the Elsevier Bibliographic databases. The manuscript management system is completely online and includes a very quick and fair peer-review system, which is all easy to use. Visit http://www.dovepress.com/ testimonials.php to read real quotes from published authors. 